Im Portrait: Wanzek Dental-Reparaturen-Schnelldienst

\section{Technik bewegt...}

\author{
Von der Kooperation zwischen der Dynadent Service für Zahnärzte GmbH und der Wanzek Den- \\ tal-Reparaturen-Schnelldienst GmbH profitieren schon seit 2009 viele hundert Mitglieder des \\ Freien Verbandes Deutscher Zahnärzte.
}

Mit ihrer zertifizierten Meisterwerkstatt führt Wanzek am Standort Mönchengladbach mit zwölf exzellent ausgebildeten Technikern jährlich bis zu 25.000 Wartungen und Reparaturen an zahnmedizinischen und labortechnischen Geräten durch. Das Unternehmen ist autorisierter Partner der führenden Dentalgerätehersteller. Die DFZ-Redaktion sprach mit dem Geschäftsführer und Inhaber Dominik Rödiger.

DFZ: Herr Rödiger, ganz direkt: Was ist das Geheimnis Ihres Erfolgs?

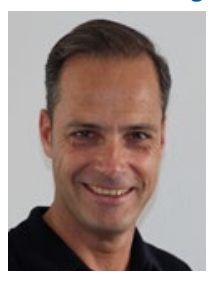
Rödiger: Basis ist die gleichbleibend hohe Qualität unserer Arbeit und die direkte und persönliche Verbindung zum Kunden. Dazu braucht es gute Mitarbeiter und Kontinuität im Personalbestand. Viele unserer Mitarbeiter sind seit über 20 Jahren im Unternehmen, bilden ein eingespieltes Team und sind für unsere Kunden jederzeit ansprechbar. Übrigens: Bereits seit 1996 arbeiten wir mit einem zertifizierten QM-System.

DFZ: Das kann noch nicht alles sein, was den Erfolg ausmacht. Rödiger: Das stimmt. Hinzu kommt die vollkommene Ausrichtung auf die Bedürfnisse des Kunden. Nicht unsere Meinung ist wichtig, sondern die Erkenntnis der Wünsche unserer Kunden zählt.

DFZ: Wie sehen die Bedürfnisse Ihrer Kunden konkret aus?

Rödiger: Betriebswirtschaftlich gesprochen: Unsere Kunden brauchen Ihre Geräte, um ihre Umsätze zu generieren. Sie müssen sich auf ihren Instrumentenpark verlassen können. Eine Wartung oder Reparatur hat daher präzise, schnell und reibungslos zu funktionieren, um die Ausfallzeiten auf ein Minimum zu reduzieren. Basis der Partnerschaft soll Vertrauen sein.

\section{DFZ: Wie setzen Sie das in der Praxis um?}

Rödiger: Wir sind serviceorientiert und reaktionsschnell. So kümmern wir uns um die Abholung der defekten Instrumente. Das Gerät wird am Tag der Zustellung repariert beziehungsweise gewartet und trifft am folgenden Tag bis zwölf Uhr bereits

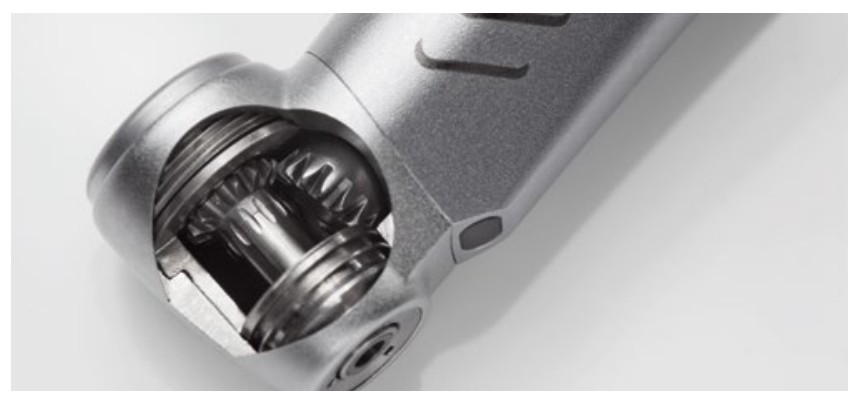

wieder beim Kunden ein. Dazu stellen wir kostenlos Mehrwegverpackungen, Versandunterlagen und Abholetiketten für den nächsten Transport zur Verfügung. So können wir einen funktionierenden 24-Stunden-Service bereitstellen.

\section{DFZ: Und die Qualitätssicherung?}

Rödiger: Für jedes Instrument, jeden Motor etc. erstellen wir eine lückenlose, jederzeit abrufbare Dokumentation. In Kürze wird der Kunde über unsere Homepage online auf seine Reparaturstatistik zugreifen können. Hiermit unterstützen wir das QM-System in der Praxis, da wir die komplette Gerätedokumentation übernehmen können. Dazu bekommt der Kunde einen Reparatur- und Garantiepass für jedes seiner Geräte. Neben praktischen Tipps für die richtige Pflege seiner Hand- und Winkelstücke geben wir auch Empfehlungen für Neuanschaffungen, wenn der Instrumentenpark aufgestockt werden soll oder die Reparatur unwirtschaftlich ist.

\section{DFZ: Sie machen es Ihren Kunden wirklich leicht.}

Rödiger: Ja, diese Transparenz ist uns wichtig und schafft Vertrauen. Wenn Sie einen Kostenvoranschlag wünschen, ist dieser stets kostenfrei - auch wenn Sie uns nicht beauftragen. Bei Wanzek sind alle Kosten für die Wartung stets einsehbar. Detaillierte Einzelauflistungen aller eingesetzten Ersatzteile mit der Original-Artikelnummer des Herstellers und die Rücksendung aller defekten Altteile gehören bei uns seit 30 Jahren zum Standard.

DFZ: Was hat darüber hinaus das FVDZ-Mitglied für Vorteile in Ihrem Haus?

Rödiger: Neben der garantiert kostenfreien Erstellung von Kostenvoranschlägen bekommen FVDZ-Mitglieder zehn Prozent Rabatt auf die gesamte Reparatur. Für Neugeräte können wir FVDZ-Mitgliedern je nach Hersteller Nachlässe bis zu 40 Prozent bieten. Aber wie schon erwähnt, die Beratung, auch zur Wahl des richtigen Instrumentes, ist uns sehr wichtig und Teil des Service-Paketes im Haus. So soll es auch in Zukunft bleiben. Herr Rödiger, wir danken Ihnen für das Gespräch.

Bei Interesse klicken Sie auf $\boldsymbol{w} w \boldsymbol{w} . f v d z . d e$ in den Bereich FVDZ Ökonomie und gehen Sie im Dynadent/Bestellcenter zum Reparatur-Schnelldienst. Dort erhalten Sie weitere Informationen.

IDS Aktion - exklusiv für Mitglieder des FVDZ!

Holen Sie sich Ihren persönlichen Reparatur-Gutschein der Firma Wanzek in Höhe von 30 Euro auf der Internationalen Dental-Schau (IDS) 2017 am Stand des FVDZ (Verteilerebene 10/11 V1) ab. 